\title{
The Effect of Pepsin Digestion on Type II Collagen Monomers
}

Jayan Rammohan and Steven J. Eppell

Department of Biomedical Engineering, Case Western Reserve University, Cleveland, OH, 441067207

Osteoarthritis is the most common degenerative bone disorder in the world today. In order to design better treatments for osteoarthritic patients, it is essential that we have a profound understanding of the molecular-scale etiology of this disease. The primary structural component of cartilage is type II collagen. By comparing pepsin-digested type II collagen monomers to intact type II collagen monomers, we gain an understanding of how the primary structural component of cartilage is affected by protease degradation. These results can provide insight to how osteoarthritic cartilage may change structurally during disease progression at the molecular level.

Type II collagen monomers were isolated from bovine cartilage and purified in two ways, creating two sample populations: (1) 51 molecules purified without the use of pepsin ("intact" type II collagen) and (2) 34 molecules purified with pepsin digestion ("pepsinized" type II collagen). Each population was imaged separately using identical conditions. A $5 \mu l$ drop of the proteins in $10 \mathrm{mM}$ acetic acid solution was deposited on freshly cleaved muscovite mica and allowed to dry. The samples were imaged by a Nanoscope III atomic force microscope (Veeco, Santa Barbara, CA) using silicon tips (Figure 1). The tips were characterized immediately preceding and immediately following each experiment. Image processing consisted of zero-order flattening and removal of artifacts resulting from tip shape using mathematical morphology.

A generalized cylinder model was used to approximate the physical dimensions of each molecule based on the experimental data. This model is ideal for fibrillar proteins such as type II collagen monomers because it uses a spline (parametric curve) to describe the backbone of each molecule as well as circular cross sections with varying radii to describe height and width data.

The average length of the intact molecules was $251+/-29 \mathrm{~nm}$ while the average length of the pepsinized molecules was $213+/-37 \mathrm{~nm}$. The average curvature of each molecule was calculated using the following formula [1]:

$$
\kappa(t)=\frac{\left|x^{\prime} y^{\prime \prime}-y^{\prime} x^{\prime \prime}\right|}{\left[\left(x^{\prime}\right)^{2}+\left(y^{\prime}\right)^{2}\right]^{3 / 2}},
$$

where $\kappa(t)$ is curvature, and $x(t)$ and $y(t)$ are functions describing the contour of the molecule as a function of contour length $\mathrm{t}$. The average curvatures of the intact and pepsinized molecules were $0.04+/-0.07 \mathrm{~nm}^{-1}$ and $0.08+/-0.08 \mathrm{~nm}^{-1}$ respectively.

Judging from the differences in length and curvature data of the intact and pepsinized populations, the results suggest that pepsin digestion of type II collagen results in shorter, more flexible monomers. However, due to the large range in length and curvature measurements in both populations, definitive conclusions regarding change in length and curvature due to pepsin digestion 
are difficult to draw. Since the primary sequence of type II collagen is known as well as the specific amino acid residue sequences on which pepsin acts, it should be possible to predict sites at which cleavage or partial cleavage of the molecule should take place by correlating residue numbers with physical length.

[1] S.I. Grossman. Calculus. Orlando, Harcourt Brace College Publishers: 821, 1992.

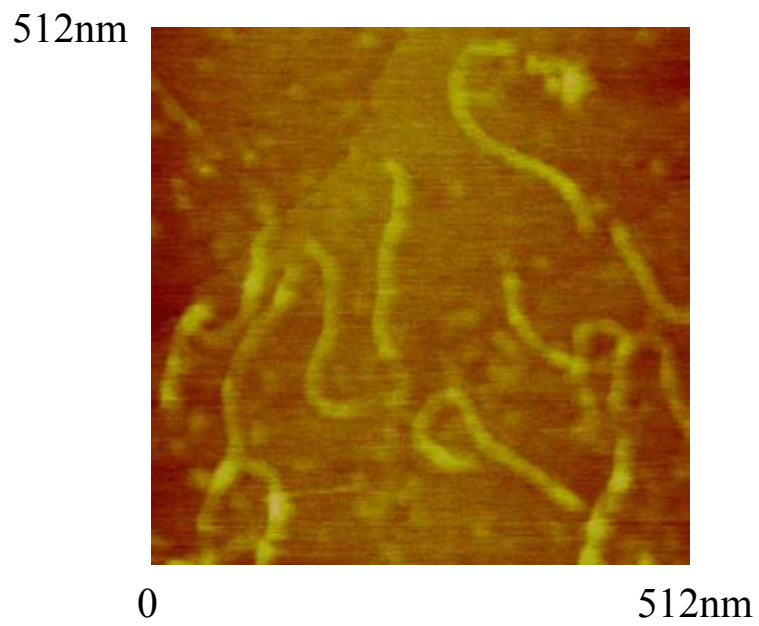

Figure 1. Type II collagen monomers deposited on mica as imaged by atomic force microscopy. Height is indicated by color with dark $(0 \mathrm{~nm})$ and light $(5 \mathrm{~nm})$.

A
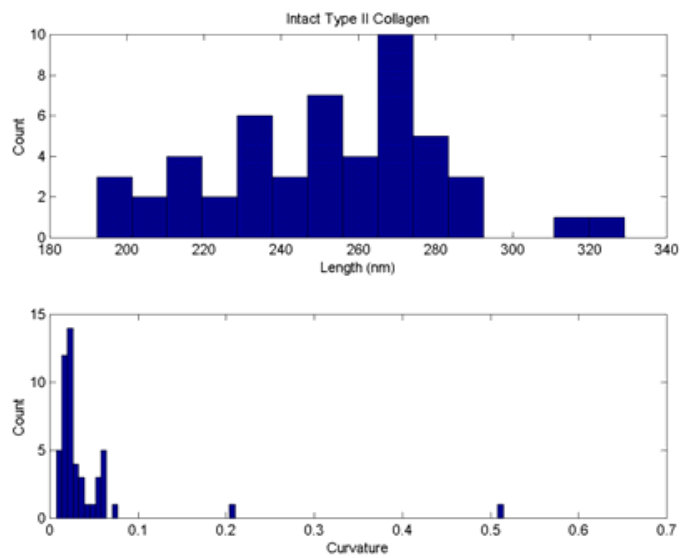

B
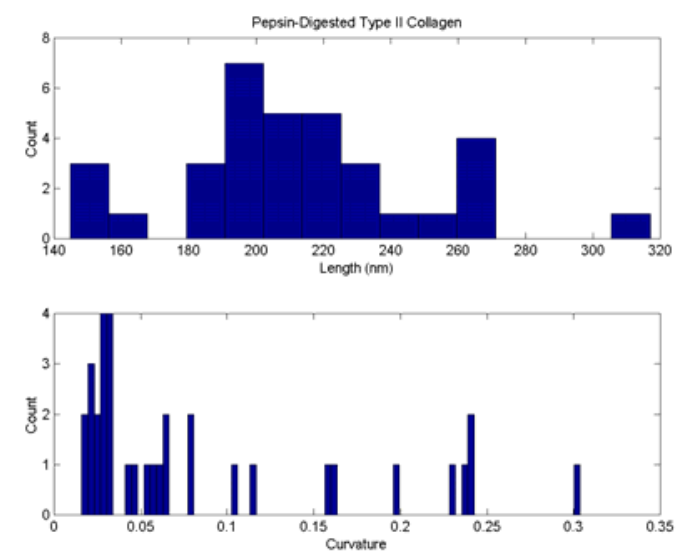

Figure 2. Histograms illustrating the length and curvature both type II collagen populations.

(A) The intact CII population had a mean length of $252+/-29 \mathrm{~nm}$. These molecules had a mean curvature of $0.04+/-0.07 \mathrm{~nm}^{-1}$.

(B) (B) The pepsin-digested CII population had a mean length of $213+/-37 \mathrm{~nm}$. These molecules had a mean curvature of $0.08+/-0.08 \mathrm{~nm}^{-1}$. 